\title{
Effect of zigzag and armchair edges on the electronic transport in single-layer and bilayer graphene nanoribbons with defects
}

\author{
Anna Orlof, J Ruseckas and Igor Zozoulenko
}

\section{Linköping University Post Print}

\section{Tweet}

N.B.: When citing this work, cite the original article.

Original Publication:

Anna Orlof, J Ruseckas and Igor Zozoulenko, Effect of zigzag and armchair edges on the electronic transport in single-layer and bilayer graphene nanoribbons with defects, 2013, Physical Review B. Condensed Matter and Materials Physics, (88), 12.

http://dx.doi.org/10.1103/PhysRevB.88.125409

Copyright: American Physical Society http://www.aps.org/ 


\title{
Effect of zigzag and armchair edges on the electronic transport in single-layer and bilayer graphene nanoribbons with defects
}

\author{
A. Orlof* \\ Mathematics and Applied Mathematics, MAI, Linköping University, SE-581 83 Linköping, Sweden \\ J. Ruseckas ${ }^{\dagger}$ \\ Institute of Theoretical Physics and Astronomy, Vilnius University, A. Goštauto 12, LT-01108 Vilnius, Lithuania \\ I. V. Zozoulenko \\ Laboratory of Organic Electronics, ITN, Linköping University, SE-601 74 Norrköping, Sweden
}

(Received 25 June 2013; revised manuscript received 21 August 2013; published 4 September 2013)

\begin{abstract}
We study electronic transport in monolayer and bilayer graphene with single and many short-range defects focusing on the role of edge termination (zigzag versus armchair). Within the tight-binding approximation, we derive analytical expressions for the transmission amplitude in monolayer graphene nanoribbons with a single short-range defect. The analytical calculations are complemented by exact numerical transport calculations for monolayer and bilayer graphene nanoribbons with a single and many short-range defects and edge disorder. We find that for the case of the zigzag edge termination, both monolayer and bilayer nanoribbons in a single- and few-mode regime remain practically insensitive to defects situated close to the edges. In contrast, the transmission of both armchair monolayer and bilayer nanoribbons is strongly affected by even a small edge defect concentration. This behavior is related to the effective boundary condition at the edges, which, respectively, does not and does couple valleys for zigzag and armchair nanoribbons. In the many-mode regime and for sufficiently high defect concentration, the difference of the transmission between armchair and zigzag nanoribbons diminishes. We also study resonant features (Fano resonances) in monolayer and bilayer nanoribbons in a single-mode regime with a short-range defect. We discuss in detail how an interplay between the defect's position at different sublattices in the ribbons, the defect's distance to the edge, and the structure of the extended states in ribbons with different edge termination influence the width and the energy of Fano resonances.
\end{abstract}

DOI: 10.1103/PhysRevB.88.125409

PACS number(s): 81.05.ue, 72.80.Vp

\section{INTRODUCTION}

Ever since the experimental isolation of graphene in 2004, ${ }^{1}$ significant research efforts have been focused on investigating the electronic and transport properties of its nanoribbons. A number of various techniques have been developed in order to fabricate graphene nanoribbons (GNRs). These include electron beam lithography and etching, ${ }^{2,3}$ chemical synthesis, ${ }^{4}$ unzipping of carbon nanotubes to form graphene nanoribbons, ${ }^{5}$ bottom-up approaches, ${ }^{6}$ and others (various aspects of fabrication and characterization of graphene nanoribbons are addressed in a recent review ${ }^{7}$ ). Special attention, both experimental and theoretical, has been paid to investigating the effect of disorder on transport in GNRs. A pronounced feature of the majority of transport experiments in GNRs is the absence of conductance quantization, ${ }^{2,3,8}$ the effect which is routinely observed in conventional semiconductor quantum wires and quantum point contact systems. ${ }^{9}$ Another distinct feature of the conductance of GNRs is the appearance of the transport gap not predicted by the transport calculations for ideal GNRs. ${ }^{2,3}$ These features in the conductance of realistic GNRs are related to the effect of disorder, such as edge disorder as well as short- and long-range disorder due to adatoms, vacancies, and defects, Coulomb impurities in substrates, ripples on the surface, etc. Theoretically, the effect of disorder in GNRs has been studied in many different contexts, including the transport gap formation, suppression of quantization, symmetries, localization, Fano resonances, and many others. ${ }^{10-23}$
Edge disorder plays an especially important role in GNRs. This is because such disorder is almost unavoidable in GNRs produced by most fabrication methods used today (especially by the commonly used etching technique), whereas the effect of disorder in the bulk of GNRs, such as charged Coulomb impurities, can be reduced by using suspended samples. ${ }^{24}$ (Note that vacancies and defects have a rather small concentration in high-quality exfoliated samples. ${ }^{25}$ ) It has been demonstrated previously that edge disorder is largely responsible for the formation of the transport gap and the suppression of the conductance quantization. ${ }^{14-16}$ Considering the importance of edge disorder for transport properties of GNRs, a question arises as to whether for a similar disorder density the nanoribbons with different edge character [zigzag and armchair (zGNR and aGNR)] are affected in a similar way or not. This is the central question in the present work. Note that the role and the manifestation of the edge character in transport and electronic properties of GNRs have been addressed in a number of previous studies. For example, zigzag and armchair edges probed by scanning tunneling spectroscopy exhibit different features of the standing wave patterns. ${ }^{26-28}$ It has also been shown that in a single-mode regime, zGNRs show the perfect conductance if the scattering is limited to long-range impurities only. ${ }^{29,30}$ This remarkable property of zGNR was attributed to the single-valley transport caused by the existence of a chiral mode propagating at the edge of the zGNR. ${ }^{31}$ The aGNRs and zGNRs also show different profiles of current distribution. ${ }^{32}$ 
In the present paper, we study the effect of edge disorder on the transport properties of both GNRs and bilayer graphene nanoribbons (BGNs), focusing on the difference between zigzag and armchair edges. We extend our calculation to the multimode regime, thus not limiting ourselves to a single-mode propagation. In contrast to previous studies, which mostly relied on numerical calculations, in the present study we develop an analytical approach that provides the exact results for the transmission coefficient of GNRs. Also, in addition to monolayer nanoribbons, we consider the case of bilayer graphene nanoribbons, with both zigzag and armchair edges (aBGNs and zBGNs). One of our most important findings is that for the case of the zigzag edge, both monolayer and bilayer nanoribbons (zGNRs and zBGNs) remain practically insensitive to the disorder situated close to the edges. This remarkable behavior is not related to the chiral edge state residing at the zigzag boundary, ${ }^{31}$ as this behavior persists into the few-mode regime as well. Instead, it is related to the effective boundary condition at the zigzag edge which does not couple valleys, ${ }^{33}$ thus hindering the intervalley scattering due to the edge disorder. (Note that the edge defects are essentially short-range scatterers favoring large momentum transfer leading to the intervalley scattering..$^{20,29,34}$ ) In contrast, the armchair edge mixes the valleys ${ }^{33}$; as a result, the conductance of both aGNRs and aBGNs is strongly affected by even a small defect concentration. We demonstrate that for a sufficiently high concentration of disorder, the difference in the conductance of zigzag and armchair ribbons diminishes in a many-mode regime. In our paper, we also address Fano resonances in a single-mode regime that originate from the interference of an extended scattering state in nanoribbons and a quasilocalized state on the defect. ${ }^{21-23}$ We discuss in detail how an interplay between the defect's position at different sublattices in the ribbons, the defect's distance to the edge, and the structure of the extended states of ribbons with different edge termination influence the width and the energy of Fano resonances.

The paper is organized as follows. In Sec. II we present the tight-binding model of $p$-orbital electrons in monolayer and bilayer nanoribbons with edge disorder. The conductance calculations in graphene nanoribbons with edge defects are performed on the basis of the recursive Green's function technique, ${ }^{35}$ which is briefly presented in Sec. II A. To analyze the conductance, we also perform calculation for a nanoribbon with a single defect situated at different distances from the edge. Such calculations are performed analytically with the help of the Green's function and the Dyson equations and are described in Sec. II B. Note that analytical calculations are especially instrumental for the case of Fano resonances, which can be quite narrow in wide nanoribbons and thus are easily missed in numerical calculations. Sections III A and IIIC present the results and a discussion concerning the effect of edge defects on the transmission of nanoribbons, and Sec. III B addresses the resonance scattering leading to Fano resonances in the conductance. Finally, Sec. IV presents the conclusions.

\section{FORMULATION AND APPROACH TO THE PROBLEM}

\section{A. Model and technique description}

In this section, we formulate the basics of electron transmission in single- and bilayer graphene nanoribbons. We use the standard nearest-neighbor $p$-orbital electron tight-binding Hamiltonian of the form $H=H_{0}+V_{0}$, where $H_{0}$ is the kinetic energy operator and $V_{0}$ describes the electron scattering on defects. We start with monolayer graphene with ${ }^{36}$

$$
H_{0}=-t \sum_{i}\left(a_{i}^{\dagger} b_{i+\Delta}+\text { H.c. }\right),
$$

where the summation runs over a hexagonal graphene lattice [Fig. 1(a)], $a_{i}^{\dagger}\left(a_{i}\right)$ and $b_{i}^{\dagger}\left(b_{i}\right)$ are the standard creation (annihilation) operators at sublattices $A$ and $B$ in the unit cell $i$ of position $p \mathbf{a}_{1}+q \mathbf{a}_{2}$ ( $p, q$ integers), and $\Delta$ denotes the nearest-neighbor cells. The parameter $t$ is the nearest-neighbor hopping energy $(t \approx 2.8 \mathrm{eV})$. The scattering on defects is described by the potential $V_{0}$,

$$
V_{0}=U \sum_{i}\left(a_{i}^{\dagger} a_{i}+b_{i}^{\dagger} b_{i}\right),
$$

where the summation runs over defected sites with the on-site potential $U$.

Bilayer graphene is considered in the form of Bernal stacking; see Fig. 1(d). The kinetic energy operator has the (a)

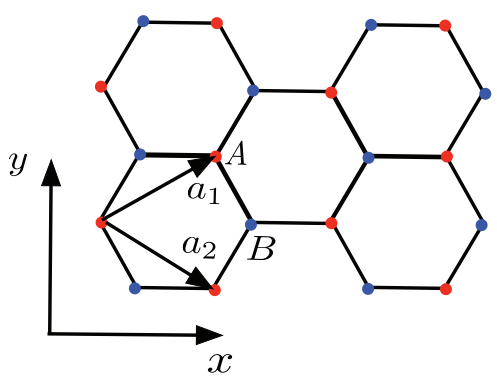

(b)

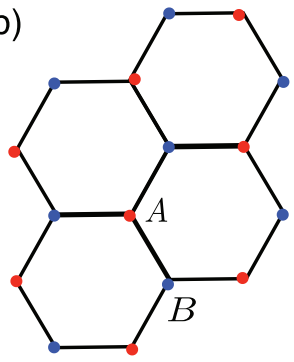

(c)

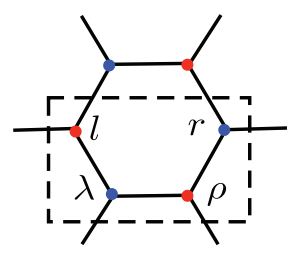

(d)

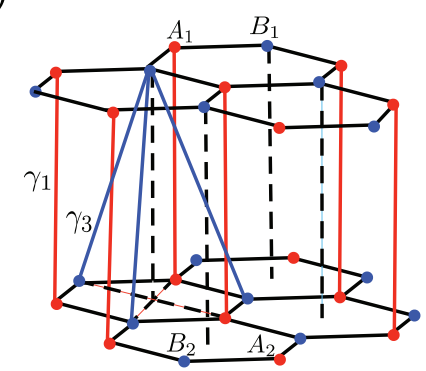

FIG. 1. (Color online) (a) Honeycomb lattice structure of graphene composed of two interpenetrating triangular lattices; $\mathbf{a}_{1}=a(3 / 2, \sqrt{3} / 2)$ and $\mathbf{a}_{2}=a(3 / 2,-\sqrt{3} / 2)$ are the lattice unit vectors, and $a \approx 1.42 \AA$ is the carbon-carbon distance. ${ }^{36} A$ (red) and $B$ (blue) mark two sublattices of the graphene lattice. (b) Asymmetric graphene sheet. (c) Labeling of carbon atoms in the rectangular unit cell used in analytical calculations. (d) Structure of bilayer graphene indicating sublattices $A_{1}, B_{1}$ (upper layer) and $A_{2}, B_{2}$ (lower layer) and hopping integrals $\gamma_{1}$ between sites in the sublattices $A_{1}$ and $A_{2}$, and $\gamma_{3}$ between sites in the sublattices $B_{1}$ and $B_{2}$. 


$$
\begin{aligned}
& \text { form }^{36,37} \\
& \qquad \begin{aligned}
H_{0}= & -\gamma_{0} \sum_{i: l=1,2}\left(a_{l, i}^{\dagger} b_{l, i+\Delta}+\text { H.c. }\right)-\gamma_{1} \sum_{i}\left(a_{1, i}^{\dagger} a_{2, i}+\text { H.c. }\right) \\
& -\gamma_{3} \sum_{i}\left(b_{1, i}^{\dagger} b_{2, i}+\text { H.c. }\right),
\end{aligned}
\end{aligned}
$$

where $a_{l, i}^{\dagger}\left(a_{l, i}\right)$ and $b_{l, i}^{\dagger}\left(b_{l, i}\right)$ are the creation (annihilation) operators for sublattices $A$ and $B$ in the layer $l=1,2$ in the unit cell $i . \gamma_{0}$ is the nearest-neighbor hopping energy within one layer $\left(\gamma_{0} \approx 3.16 \mathrm{eV}\right)$. In calculations for BGNs we assume $\gamma_{1}=\frac{0.39}{3.16}\left[\gamma_{0}\right]$ and $\gamma_{3}=0$, i.e., we consider the minimal low-energy model. ${ }^{36,37}$ A definition of sublattices for bilayer graphene is illustrated in Fig. 1: sites belonging to the sublattices $A_{1}$ and $A_{2}$ are situated on the top of each other, whereas sites belonging to $B_{1}$ and $B_{2}$ are displaced. The scattering on defects is described by

$$
V_{0}=U \sum_{i: l=1,2}\left(a_{l, i}^{\dagger} a_{l, i}+b_{l, i}^{\dagger} b_{l, i}\right)
$$

where the summation runs over defected sites with the on-site potential $U$. From now on we will express all energies in the units of the hopping energy $t$ for monolayer graphene and $\gamma_{0}$ for bilayer graphene. In our calculation, we use a model of the strong short-range scattering setting $U=100$ in numerical calculations and setting $U \rightarrow \infty$ in analytical ones. This model is appropriate to describe the absence of a carbon atom at the edge of a nanoribbon or a vacancy in the bulk, as well as to describe a scattering on an adatom. ${ }^{38,39}$

The conductance calculation of monolayer graphene nanoribbons (GNRs) with a single defect is performed analytically and confirmed numerically. The analytical approach is described in detail in the next section. The calculation of the conductance in monolayer graphene nanoribbons with many defects and all calculations for bilayer graphene nanoribbons (BGNs) are performed numerically on the basis of the recursive Green's function technique. ${ }^{18,35}$ In this technique, a ribbon of width $W$ is divided into three regions, namely a left lead, a scattering region, and a right lead. The scattering potential is defined in the scattering region of length $L$, whereas both semi-infinite leads are considered to be ideal (no scattering). In the recursive Green's function technique, Green's functions of every slice in the scattering region are calculated and recursively coupled by the Dyson equation to obtain the Green's function of the whole scattering region. The surface Green's function of the leads is calculated using the wave functions of the Bloch states of the infinite leads. The transmission and reflection amplitudes are obtained with use of previously calculated Green's functions. The connection between the transmission and the conductance at zero temperature is provided by the Landauer formula,

$$
G=\frac{2 e^{2}}{\hbar} \sum_{\alpha, \beta} T_{\beta, \alpha}, T_{\beta, \alpha}=\left|t_{\beta, \alpha}\right|^{2},
$$

where $T_{\beta, \alpha}$ and $t_{\beta, \alpha}$ are, respectively, the transmission coefficient and the transmission amplitude from the incoming state $\alpha$ in the left lead to the outgoing state $\beta$ in the right lead. We also calculate the local density of states (LDOS) of graphene nanoribbons with a defect, expressing it via the imaginary part of the Green's function of the ribbon in a standard way. ${ }^{18,35}$

\section{B. Analytical expressions for transmission of electrons in monolayer graphene nanoribbons with a single defect}

For configurations of graphene with rectangular geometry, it is convenient to use a rectangular unit cell, as has been done in Refs. 40 and 41. Such a unit cell has four atoms labeled with the symbols $l, \lambda, \rho$, and $r$, as shown in Fig. 1(c). The atoms with labels $l$ and $\rho$ belong to sublattice $A$, and the atoms with labels $\lambda$ and $r$ belong to sublattice $B$. We use dimensionless Cartesian components of the wave vector

$$
\kappa=3 a k_{x}, \quad \xi=\sqrt{3} a k_{y}
$$

instead of the wave-vector components $k_{x}$ and $k_{y}$. The first Brillouin zone corresponding to the rectangular unit cell contains the values of the wave vectors $\kappa$ and $\xi$ in the intervals $-\pi \leqslant \kappa<\pi,-\pi \leqslant \xi<\pi$. Compared to the area of the Brillouin zone of the hexagonal unit cell, the area of the Brillouin zone of the rectangular unit cell is two times smaller. The smaller Brillouin zone leads to the appearance of additional dispersion branches. Those dispersion branches can be taken into account by using the values of the wave vector in the longitudinal direction from a two times larger interval $[-2 \pi, 2 \pi)$.

Analytical expressions for wave functions in graphene nanoribbons were provided in Refs. 40 and 41. Eigenfunctions of tight-binding Hamiltonian (1) in armchair and zigzag graphene nanoribbons can be written as

$$
\psi_{\nu, \kappa_{\|}}^{\sigma}(\ell)=\chi_{\nu, \kappa_{\|}}^{\sigma}\left(\ell_{\perp}\right) e^{i \kappa_{\|} m} .
$$

Here $\sigma=\mathrm{aGNR}, \mathrm{zGNR}$, and the triplet $\ell=m, n, \alpha$ indicates the position of the site. The numbers $m$ and $n$ determine the position of the rectangular unit cell and $\alpha=l, \rho, \lambda, r$ shows the position in the cell. For aGNR, the transverse and longitudinal components of the wave vector are $\kappa_{\perp}=\xi$, $\kappa_{\|}=\kappa$, and for zGNR they are $\kappa_{\perp}=\kappa, \kappa_{\|}=\xi$. The functions $\chi_{v, \kappa_{\|}}^{\sigma}\left(\ell_{\perp}\right)$ with $\ell_{\perp}=n, \alpha$ are transverse mode wave functions. The corresponding eigenenergies are

$$
E(\kappa, \xi)=s_{1}|\phi(\kappa, \xi)|
$$

with $s_{1}= \pm 1$ and

$$
\phi(\kappa, \xi)=-e^{-i \frac{\kappa}{2}}+2 \cos \left(\frac{\xi}{2}\right) .
$$

The square of the absolute value of $\phi(\kappa, \xi)$ is

$$
|\phi(\kappa, \xi)|^{2}=1+4 \cos ^{2}\left(\frac{\xi}{2}\right)-4 \cos \left(\frac{\xi}{2}\right) \cos \left(\frac{\kappa}{2}\right) .
$$

The longitudinal component of the wave vector $\kappa_{\|}$can take values from the interval $-2 \pi \leqslant \kappa_{\|}<2 \pi$. The possible values of the transverse component of the wave vector are determined by the boundary conditions. In tight-binding calculations, there is a difference between graphene nanoribbons having the longitudinal axis of symmetry and without it. A symmetrical sheet of graphene is shown in Fig. 1(a), whereas an asymmetrical sheet is shown in Fig. 1(b). We consider graphene nanoribbons having $N$ whole rectangular unit cells in 
the transverse direction. That is, zGNRs with the longitudinal axis of symmetry and aGNRs without the axis of symmetry have $N$ rectangular unit cells in the transverse direction, while zGNRs without the axis of symmetry and aGNRs with the longitudinal axis of symmetry have $N+1 / 2$ rectangular unit cells in the transverse direction. From now on, we will write equations only for symmetrical nanoribbons. Equations for asymmetrical aGNRs can be obtained by changing the number $N$ to $N-1 / 2$ and for asymmetrical zGNRs by changing to $N+1 / 2$.

For aGNR, the transverse component of the wave vector is

$$
\xi_{v}=\frac{\pi v}{N+1}, \quad v=1, \ldots, N+1,
$$

and for $z$ GNR the transverse component of the wave vector $\kappa_{v} \equiv \kappa_{v}(\xi)$ is the solution of the equation

$$
\frac{\sin \left(\kappa_{\nu} N\right)}{\sin \left[\kappa_{\nu}\left(N+\frac{1}{2}\right)\right]}=2 \cos \left(\frac{\xi}{2}\right), \quad v=1, \ldots, N
$$

The expressions for the transverse mode wave functions $\chi_{v, \kappa \|}^{\sigma}\left(\ell_{\perp}\right)$ are given in Appendix.

For calculation of the Green's function, it is useful to have the eigenfunctions of graphene ribbons characterized not by the longitudinal wave vector but by the energy. Given the energy, the components of the wave vector $\xi_{v}$ and $\kappa_{v}$ for aGNR can be calculated from Eq. (11) and

$$
\kappa_{v}(E)=s_{1} 2 \arccos \left[\frac{1-E^{2}}{4 \cos \left(\frac{\xi_{v}}{2}\right)}+\cos \left(\frac{\xi_{v}}{2}\right)\right] .
$$

The sign $s_{1}$ can be determined from the sign of the energy: $s_{1}=\operatorname{sgn}(E)$. For zGNR, the wave vector $\kappa_{v}^{(i)}$ is the solution of the equation

$$
|E|=\left|\frac{\sin \left(\frac{\kappa_{v}^{(i)}}{2}\right)}{\sin \left[\kappa_{v}^{(i)}\left(N+\frac{1}{2}\right)\right]}\right|
$$

whereas the wave vector $\xi_{v}^{(i)}(E)$ is determined from the equation

$$
\xi_{v}^{(i)}= \pm 2 \arccos \left(\frac{\sin \left(\kappa_{v}^{(i)} N\right)}{2 \sin \left[\kappa_{v}^{(i)}\left(N+\frac{1}{2}\right)\right]}\right) .
$$

Here index $i=1,2$ numbers solutions having the same index $v$; different indices $i$ correspond to different valleys.

Given the eigenfunctions $\psi_{v, \kappa_{\|}}^{\sigma}(\ell)$, the general expression for the retarded Green's function is

$$
G_{0}\left(\ell, \ell^{\prime} ; E\right)=\sum_{s_{1}= \pm 1} \sum_{\nu} \int_{-2 \pi}^{2 \pi} d \kappa_{\|} \frac{\psi_{\nu, \kappa_{\|}}^{\sigma}(\ell) \psi_{\nu, \kappa_{\|}}^{\sigma *}\left(\ell^{\prime}\right)}{E-E(\kappa, \xi)+i \eta}
$$

From Eq. (16) for symmetrical zGNR we obtain the retarded Green's function

$$
G_{0}\left(\ell, \ell^{\prime} ; E\right)=-i \sum_{\nu=1}^{N} \sum_{i=1,2} \frac{1}{v_{\nu, i}^{\mathrm{zGNR}}(E)} \begin{cases}\chi_{\nu, i, \xi_{v}^{(i)}}^{\mathrm{zGNR}}\left(\ell_{\perp} ; E\right) \chi_{\nu, i,-\xi_{v}^{(i)}}^{\mathrm{zGNR}}\left(\ell_{\perp}^{\prime} ; E\right) e^{i \xi_{v}^{(i)}(E)\left(m-m^{\prime}\right)}, & m>m^{\prime}, \\ \chi_{\nu, i,-\xi_{v}^{(i)}}^{\mathrm{zGNR}}\left(\ell_{\perp} ; E\right) \chi_{\nu, i, \xi_{v}^{(i)}}^{\mathrm{zGNR}}\left(\ell_{\perp}^{\prime} ; E\right) e^{-i \xi_{\nu}^{(i)}(E)\left(m-m^{\prime}\right)}, \quad m<m^{\prime},\end{cases}
$$

where

$$
v_{\nu, i}^{\mathrm{ZGNR}}=\left.\frac{\partial}{\partial \xi} E\left(\kappa_{\nu}^{(i)}(\xi), \xi\right)\right|_{\xi=\xi_{v}^{(i)}}=-s_{1}(-1)^{\nu} \frac{N \sin \left(\frac{\kappa_{v}^{(i)}}{2}\right) \cos \left[\kappa_{\nu}^{(i)}\left(N+\frac{1}{2}\right)\right]-\frac{1}{2} \sin \left(\kappa_{\nu}^{(i)} N\right)}{N \sin \left(\frac{\kappa_{v}^{(i)}}{2}\right)-\frac{1}{2} \sin \left(\kappa_{\nu}^{(i)} N\right) \cos \left[\kappa_{\nu}^{(i)}\left(N+\frac{1}{2}\right)\right]} \sin \left(\frac{\xi_{\nu}^{(i)}}{2}\right)
$$

is the velocity. The sign of $\xi_{v}^{(i)}$ in Eq. (15) should be chosen in such a way that the velocity $v_{v, i}^{\mathrm{zGNR}}$ is positive.

The symmetrical aGNR has states localized on the atoms with indices $\rho$ and $\lambda$ with the energies $E= \pm 1 .{ }^{40}$ Thus the expression for the retarded Green's function in symmetrical aGNR is

$$
\begin{aligned}
G_{0}\left(\ell, \ell^{\prime} ; E\right)= & -i \sum_{\nu=1}^{N} \frac{1}{v_{\nu}^{\mathrm{aGNR}}(E)} \begin{cases}\chi_{\nu, \kappa_{v}}^{\mathrm{aGNR}}\left(\ell_{\perp} ; E\right) \chi_{\nu,-\kappa_{v}}^{\mathrm{aGNR}}\left(\ell_{\perp}^{\prime} ; E\right) e^{i \kappa_{\nu}(E)\left(m-m^{\prime}\right)}, & m>m^{\prime} \\
\chi_{\nu,-\kappa_{v}}^{\mathrm{aGNR}}\left(\ell_{\perp} ; E\right) \chi_{\nu, \kappa_{v}}^{\mathrm{aGNR}}\left(\ell_{\perp}^{\prime} ; E\right) e^{-i \kappa_{v}(E)\left(m-m^{\prime}\right)}, & m<m^{\prime}\end{cases} \\
& +\sum_{s_{1}= \pm 1} \frac{\chi_{N+1}^{\mathrm{aGNR}}\left(\ell_{\perp} ; E\right) \chi_{N+1}^{\mathrm{aGNR} *}\left(\ell_{\perp}^{\prime} ; E\right)}{E-s_{1}} \delta_{m, m^{\prime}},
\end{aligned}
$$

where

$$
v_{v}^{\mathrm{aGNR}}=\left.\frac{\partial}{\partial \kappa} E\left(\kappa, \xi_{v}\right)\right|_{\kappa=\kappa_{v}}=\frac{1}{E} \cos \left(\frac{\xi_{v}}{2}\right) \sin \left(\frac{\kappa_{v}}{2}\right)
$$

is the velocity. The last term in Eq. (19) is from the localized states corresponding to $v=N+1$. For asymmetrical aGNR, there is no such localized states and the last term in Eq. (19) is absent.

The expression for the Green's function of a system with a single defect is ${ }^{45}$

$$
G\left(\ell, \ell^{\prime}\right)=G_{0}\left(\ell, \ell^{\prime}\right)+\frac{G_{0}\left(\ell, \ell_{0}\right) U G_{0}\left(\ell_{0}, \ell^{\prime}\right)}{1-U G_{0}\left(\ell_{0}, \ell_{0}\right)}
$$


where $G_{0}=\left[E-H_{0}+i \eta\right]^{-1}$ is the retarded Green's function of the graphene nanoribbon without defect. The transmission amplitude from the transverse mode $v^{\prime}$ to $v$ can be calculated using the Green's function ${ }^{46}$

$$
t_{\nu, v^{\prime}}=i \sqrt{v_{\nu} v_{\nu^{\prime}}} \sum_{\ell_{\perp}, \ell_{\perp}^{\prime}} \chi_{\nu}^{*}\left(\ell_{\perp}\right) e^{-i \kappa_{v}\left(m-m_{0}\right)} G\left(\ell, \ell^{\prime}\right) \chi_{v^{\prime}}\left(l_{\perp}^{\prime}\right) e^{i \kappa_{\nu^{\prime}}\left(m^{\prime}-m_{0}\right)} .
$$

Using Eqs. (19), (21), and (22), the transmission amplitude for aGNR with a defect is

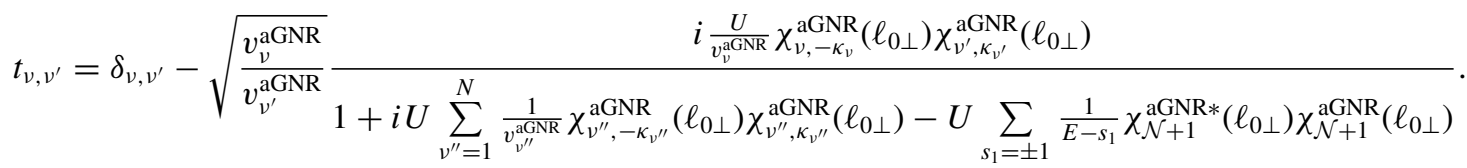

For zGNR with a defect, the transmission amplitude is

$$
t_{v, i ; v^{\prime}, i^{\prime}}=\delta_{v, v^{\prime}} \delta_{i, i^{\prime}}-\sqrt{\frac{v_{v, i}^{\mathrm{zGNR}}}{v_{v^{\prime}, i^{\prime}}^{\mathrm{zGNR}}}} \frac{i \frac{U}{v_{v, i}^{\mathrm{ZGNR}}} \chi_{\nu, i,-\xi_{v}^{(i)}}^{\mathrm{zGNR}}\left(\ell_{0 \perp}\right) \chi_{v^{\prime}, i^{\prime}, \xi_{v^{\prime}}^{\left(i^{\prime}\right)}}^{\mathrm{zGNR}}\left(\ell_{0 \perp}\right)}{1+i U \sum_{v^{\prime \prime}=1}^{N} \sum_{i^{\prime \prime}} \frac{1}{v_{v^{\prime \prime}, i^{\prime \prime}}^{\mathrm{ZGN}}} \chi_{v^{\prime \prime}, i^{\prime \prime},-\xi_{v^{\prime \prime}}^{\left(i^{\prime \prime}\right)}}^{\mathrm{zGNR}}\left(\ell_{0 \perp}\right) \chi_{v^{\prime \prime}, i^{\prime \prime}, \xi_{v^{\prime \prime}}^{\left(i^{\prime \prime}\right)}}^{\mathrm{zGNR}}\left(\ell_{0 \perp}\right)} .
$$

If the defect is an absence of an atom, then Eqs. (23) and (24) should be taken in the limit $U \rightarrow \infty$. From this point on we will consider such defects.

\section{CONDUCTANCE OF MONOLAYER GRAPHENE NANORIBBONS AND BILAYER GRAPHENE NANORIBBONS WITH DEFECTS}

In this section, we present and discuss the results for the conductance of nanoribbons with single and many defects. Most of the calculations are performed for zGNRs and zigzag bilayer graphene nanoribbons of width $23.71 \mathrm{~nm}$ as well as metallic aGNRs and armchair bilayer graphene nanoribbons of width $23.73 \mathrm{~nm}$, if not stated differently. (Calculations in Sec. III B are performed for narrower ribbons.)

\section{A. Effect of a single defect}

We first consider zigzag and armchair nanoribbons with a single defect at the edge. Figures 2(a) and 2(b) show the transmission of, respectively, zGNR and aGNR as a function of electron energy. There is a striking difference between the transmission curves. Namely, while the transmission of the $\mathrm{zGNR}$ remains practically unaffected by the presence of the defect, the transmission of the aGNR shows noticeable deviations from the ideal steps. Another difference is that when the defect is situated at the sublattice $B$, the transmission of the zGNR shows a narrow resonance (a dip) close to the propagation threshold for the second mode. (Resonant features in the transmission will be addressed in detail in the next subsection.)

The above features can also be seen in the transmission of BGNs; see Figs. 2(c) and 2(d). The transmission of the $\mathrm{zBGN}$ is also practically unaffected by the defect, and exhibits a narrow resonance when the defect is on sublattice $B_{1}$ or $A_{2}$. For the case of aBGNs with a defect on sublattice $B_{1}$ or $B_{2}$, the ribbon's transmission is even more suppressed in comparison to the case of aGNRs and shows a broad dip in the energy region of the first propagating mode. In contrast, the transmission of aBGNs is affected very little when a defect is on sublattice $A_{1}$ or $A_{2}$.

We now discuss how the transmission depends on the defect position across a nanoribbon. Figure 3 (left panels) shows the transmission of nanoribbons in the energy interval of the first propagating mode. We first note that the transmission of aGNRs and aBGNs for certain defect positions is practically unaffected by the presence of a defect [curves in Figs. 3(b) and 3(d) corresponding to $T=1$ ]. This is because in these positions the wave function of armchair ribbons vanishes or is very small ${ }^{40,41}$ (Sec. III B), and therefore the defect is not "felt" by the incoming states. [Note that this is the reason why the transmission in aBGNs is not affected for certain positions of a defect on the edge in Fig. 2(d).]

Let us now focus on the most important difference between armchair and zigzag nanoribbons relevant to the present study. That is, if a defect is situated sufficiently close to the edge of zGNRs and zBGNs, the transmission in the first mode, as well as in the few-mode regime, remains practically unaffected, $T \approx 1$. In contrast, for aGNRs and aBGNs the transmission drops, respectively, to $T \approx 0.85$ and $T \approx 0.7$ (except for the defect positions where the wave function is zero and hence $T=1$ ). The explanation for the different behavior of zigzag and armchair nanoribbons containing defects close to the edges can be related to the absence of the mixing of $K$ and $K^{\prime}$ valleys at the edge of an ideal zigzag ribbon. Indeed, it is know that the zigzag edges impose the boundary conditions that do not allow valley mixing, whereas the armchair edges do mix valleys. ${ }^{42-44}$ On the other hand, it has been shown that in the hexagonal lattice, short-range scatterers (considered in the present study) mix $K$ and $K^{\prime}$ valleys, ${ }^{29,34}$ and if the effective range of the scatterer is of the order of the carboncarbon distance or less, the intervalley scattering is much more efficient than the intravalley one. ${ }^{34}$

Let us first start with the single-mode propagation. In zigzag nanoribbons, the forward-propagating mode belongs to the $K$ valley, whereas the mode propagating in the opposite direction belongs to $K^{\prime}$ valley. ${ }^{29}$ Therefore, if a defect is situated close to the edge of the zigzag ribbon, the edge prevents the valley 


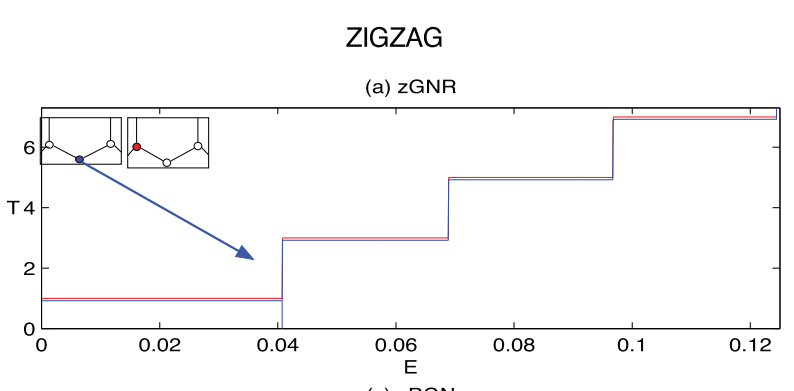

(c) zBGN

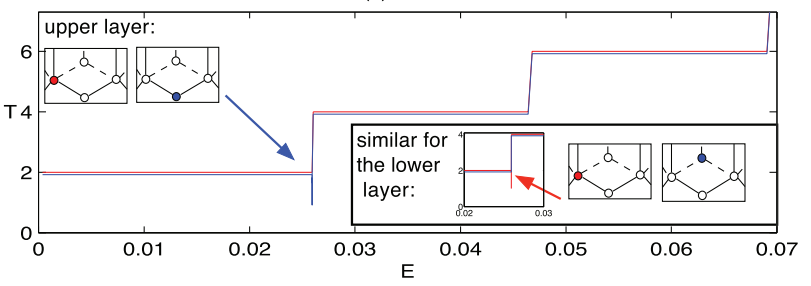

ARMCHAIR

(b) aGNR

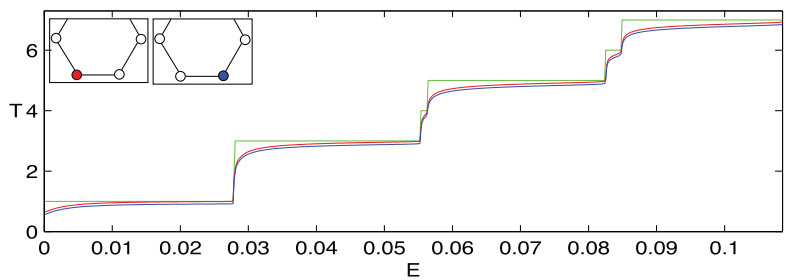

(d) $a B G N$

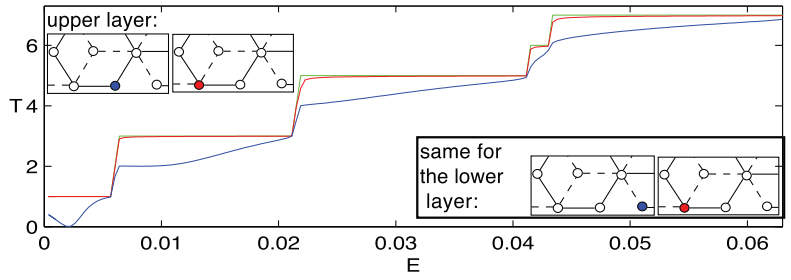

FIG. 2. (Color online) Transmission of the monolayer and bilayer nanoribbons with a single defect at the edge as a function of energy. Insets indicate defect positions. Red and blue curves correspond to a defect in sublattices $A$ and $B$, respectively. Green curves indicate the transmission probability for ideal nanoribbons (without defects). Transmission curves are slightly shifted for clarity in the vertical direction.

mixing and therefore the electron cannot be backscattered as this would require mixing of $K$ and $K^{\prime}$ valleys. As a result, the transmission remains unaffected. When the defect is moved from the ribbon's edge, the influence of the edge diminishes and the backscattering becomes possible. This is clearly seen in Fig. 3(a), where the transmission coefficient decreases from its value $T=1$ when the distance from the edge increases. For armchair nanoribbons, the edge does not prevent mixing of $K$ and $K^{\prime}$ valleys. This allows the backscattering by the shortrange defects regardless of their distance from the edge. This apparently holds true regardless of a number of propagating modes in the ribbon [see Fig. 3(b)].

Consider now a few-mode regime in zigzag nanoribbons. In this case, forward- and backward-propagating states belong to both $K$ and $K^{\prime}$ valleys. ${ }^{29}$ Nevertheless, because the intravalley scattering is much less efficient than the intervalley one, ${ }^{34}$ and the latter is suppressed at the ribbon's edge, the zigzag nanoribbons remain insensitive to the scattering by short-range defects close to the edge in a few-mode regime as well (not shown here). However, as the energy of the incoming electron increases, the efficiency of the intravalley scattering becomes comparable to that of the intervalley one. As a result, the distance to the edge when the conductance is not affected by the presence of the defect shrinks, and in the many-mode regime the transmission becomes significantly affected even for defects situated on the second row; see Fig. 3 (right panels).

\section{B. Resonant reflection in graphene nanoribbons}

In this subsection, we discuss in detail resonance features in the conductance of nanoribbons and identify them as Fano resonances. ${ }^{21-23,48}$ We first note that a single defect (a) zGNR
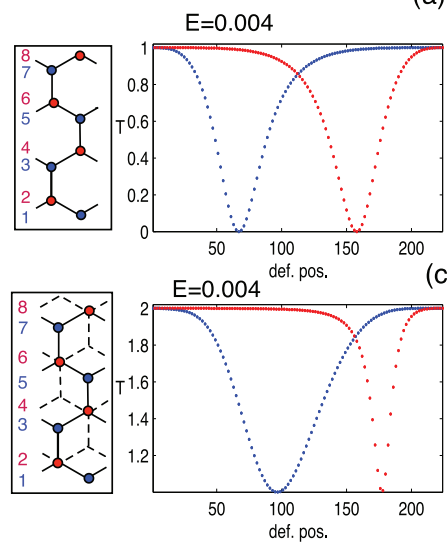

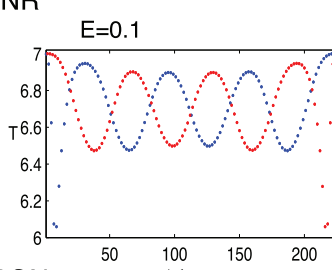

(c) $\mathrm{ZBGN}$

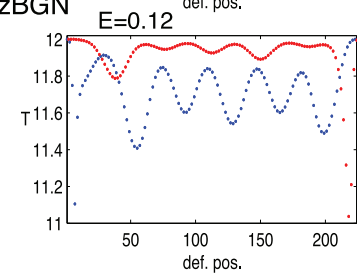

(b) aGNR
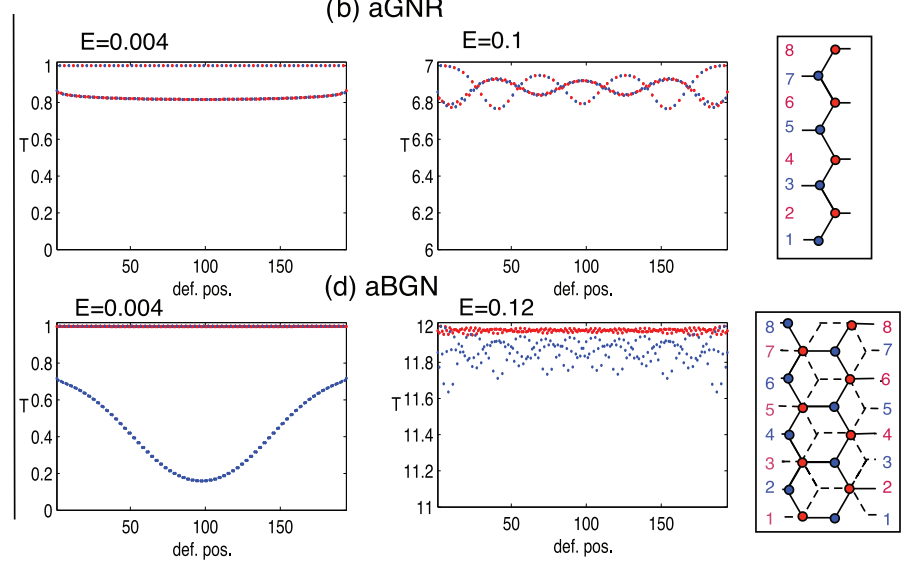

FIG. 3. (Color online) Transmission of the monolayer and bilayer nanoribbons with a single defect as a function of the defect's position across the nanoribbons in a single- and many-mode regime [left and right panels in (a) and (b)]. Red and blue curves correspond to a defect in sublattices $A$ and $B$, respectively. For bilayer nanoribbons, the defect is in the upper layer. Insets indicate the numbering of sites. 


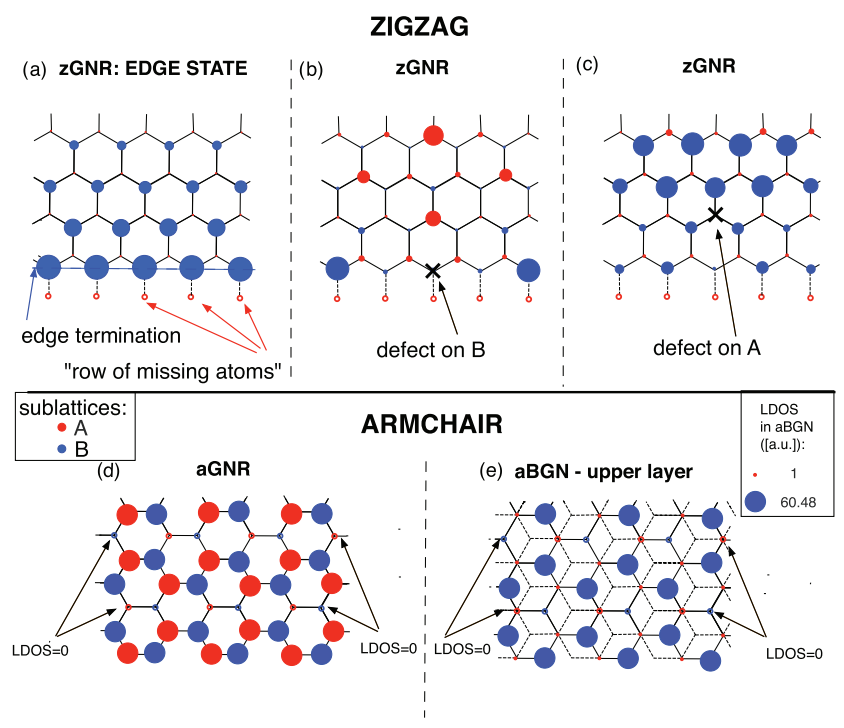

FIG. 4. (Color online) LDOS for monolayer and bilayer zigzag and armchair nanoribbons in the energy interval corresponding to the first propagating mode. (a) Ideal zGNR terminated by $B$ atoms at the lower edge. (b) A defect in sublattice $B$ at the edge of zGNR at the energy corresponding to the resonant dip. (c) A defect in sublattice $A$ close to the edge of zGNR. [The values of LDOS in (b) are $\sim 1000$ times higher in comparison to (a) and (c).] (d) Ideal aGNR; (e) Ideal aBGN (LDOS is indicated for the upper layer). Empty circles in (d) and (e) correspond to zero LDOS.

(vacancy) in an infinite graphene sheet accommodates a localized quasibound state with the energy $E=0 .{ }^{21,47}$ The wave function of this state vanishes at the sublattice hosting the defect and is localized around the defect at another sublattice. The observed resonant dips in the transmission of nanoribbons represent well-known Fano resonances ${ }^{48}$ that originate from the interference of an extended state in the ribbon with a weakly coupled quasibound state residing at the defect. The width of the resonant dip is determined by the strength of the coupling between the quasibound state and the extended state: the weaker the coupling, the narrower the dip. These types of resonances are well known in conventional two-dimensional semiconductor heterostructures. ${ }^{49-52}$ The Fano resonances in the transmission of graphene nanoribbons have been addressed in Refs. 21-23. In the present study, we provide a detailed discussion on how the width and energy of these resonances depend on the edge termination, the defect's position at different sublattices in the ribbons, and the distance to the edge for both cases of monolayer and bilayer nanoribbons.

Let us first consider a zGNR and, for the sake of concreteness, define its edge atoms as those belonging to sublattice $B$; see Fig. 4(a) for illustration. Figure 5(a) shows the transmission probability of the zGNR with a defect at sublattices $A$ or $B$ for a unit cell situated at a different distance $n$ from the edge. The transmission coefficient of the zGNR exhibits the following features. For $n=1$, the transmission shows a narrow dip (antiresonance) close to the propagation threshold of the second mode if the defect belongs to the same sublattice as the edge termination (i.e., sublattice $B$ ). If the defect belongs to sublattice $A(n=4)$, the transmission remains practically unaffected; see Fig. 5(a). When the defect at sublattice $B$ is moved toward the center of the ribbon, the antiresonance dip becomes wider and its position shifts to lower energies. At the same time, for a defect at sublattice $A$, a dip close to $E=0$ starts to develop and it becomes more pronounced as the defect is moved toward the center. Finally, when the defect is in the middle of the ribbon at sublattice $A$ or $B$, the transmission coefficient exhibits very similar features for both defect positions, showing a broad dip in the vicinity of $E=0$.

To understand these resonance features, we calculate and analyze the LDOS of a ribbon with a defect. Consider now an ideal zGNR (without defects) with the edge atoms at sublattice $B$. In the vicinity of this edge, the wave function is localized at sublattice $B$ and is negligibly small at sublattice $A$. Note that the wave function vanishes at a row of missing atoms belonging (a) zGNR
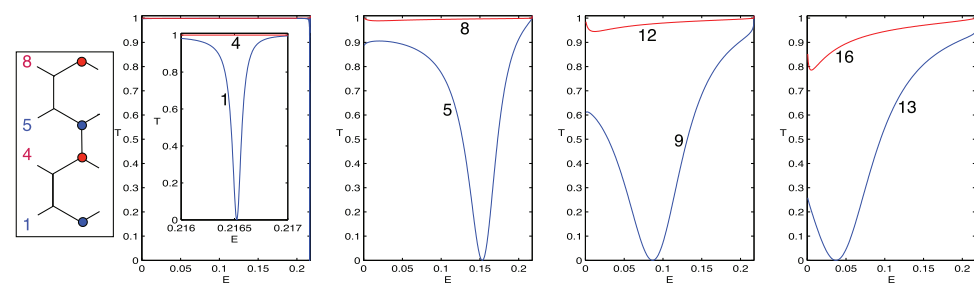

(b) zBGN
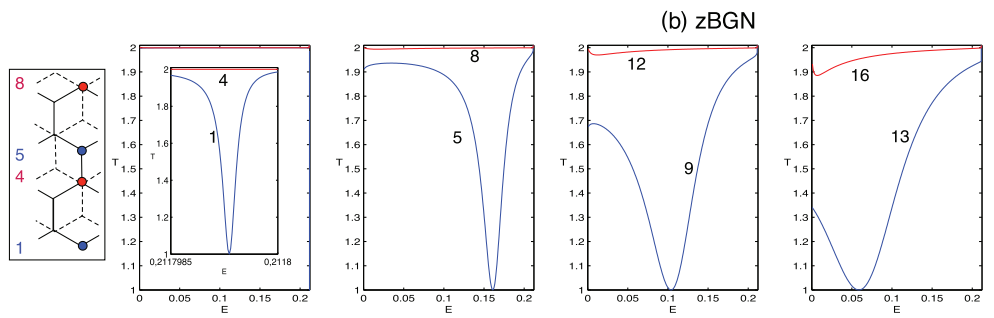
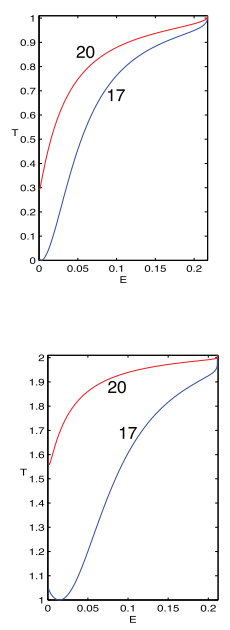

(c) aGNR
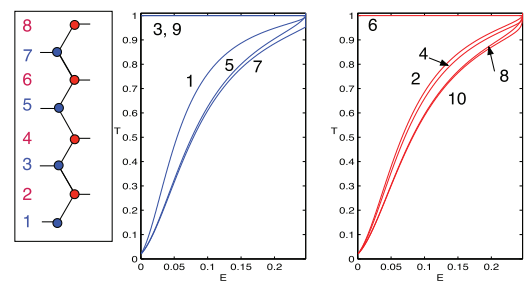

(d) aBGN
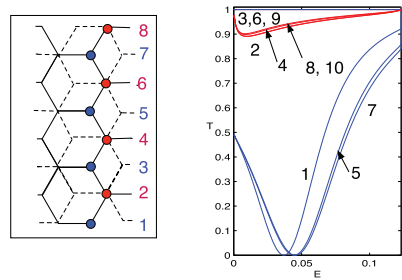

FIG. 5. (Color online) Resonant dips in the transmission of a (a) zGNR, (b) zBGN, (c) aGNR, and (d) BGN with a single defect situated at different distances from the ribbon's edge as indicated in the figures. Red and blue curves correspond to the defect on the $A$ and $B$ sublattices. The number of sites across the nanoribbons is $M=40$ for zGNR and zBGN (corresponding to the width $w=4.12 \mathrm{~nm}$ ), and $M=20$ for aGNR and aBGN (corresponding to the width $w=2.34 \mathrm{~nm}$ ). 
to sublattice $A$; see Fig. 4(a) for illustration. Consider now a defect at the edge of a zGNR on sublattice $B$ as illustrated in Fig. 4(b). The wave function of the quasibound state of such a defect is apparently strongly affected by the presence of the edge. Indeed, the resonant state cannot be localized in the immediate vicinity of the defect at sublattice $A$ because the edge strongly suppresses the wave function there, and the wave function has to vanish at the neighboring row of missing $A$ atoms. As a result, the wave function of the resonant state is strongly distorted in comparison to the case of a defect on an infinite sheet, and the resonant energy is shifted from $E=0$ to higher energies. The influence of the edge on the wave function of the resonant state decreases as the defect is moved from the edge, which leads to the shift of the resonant energy back to $E=0$ when the defect is moved toward the center of the ribbon. The calculated LDOS in a ribbon with a defect is shown in Fig. 6, which illustrates the influence of the ribbon's edge on the wave function of the localized state residing at defects situated at different distances from the edge. Let us now discuss the width of the Fano resonances. When the defect is situated at the ribbon's edge at sublattice $B$, the coupling between the extended state in the nanoribbon and the localized state at the defect is the weakest and it increases as the defect is moved toward the middle of the ribbon. This is because the wave function of the defect is localized at sublattice $A$, whereas the amplitude of the wave function of the extended state at sublattice $A$ is negligibly small at the edge and increases toward the middle; see Fig. 4(a). Hence, the overlap between the wave functions increases, which results in the increase of the width of the resonant dip; see Fig. 5(a). When the distance from the defect to the edge increases, the dip's position shifts toward $E=0$, which is related to the shift of the resonant energy due to the reduced effect of the edge on the wave function of the localized state as discussed above.

When the defect is situated at the ribbon's edge at sublattice $A$, both the defect's wave function and the wave function of an extended state are localized at sublattice $B$; see Figs. 4(a) and 4(c). As a result, the overlap and hence the coupling between the wave functions are strong and the resonant behavior is manifested as a broad dip when the transmission coefficient $T$ does not vanish. (Note that a narrow resonance requires the formation of a long-lived quasibound state, which is possible only for the case of a weak coupling between the extended and the quasibound state.) When the defect is moved from the edge, the overlap between the defect's wave function and the wave function of the extended state decreases because the amplitude of the former diminishes toward the opposite edge of the ribbon. This leads to the formation of a resonant dip at $E=0$; see Fig. 5(a). Note that in the present case of the defect on sublattice $A$, the resonant energy does not depend on the distance of the defect to the ribbon's edge. In contrast to the previous case, the ribbon's edge does not distort the wave function of the quasibound state because the missing row of atoms [where the wave function has to vanish; see Fig. 4(a)] belongs to sublattice $A$, while the wave function of the quasibound state is negligibly small anyway.

Finally, it is noteworthy that for both cases of defects on the $A$ and $B$ sublattices, the position and the width of the resonant dips are rather similar when the defects are situated in the middle of the ribbon. This is because in the center of

\section{Defect on B sublattice}

(a)
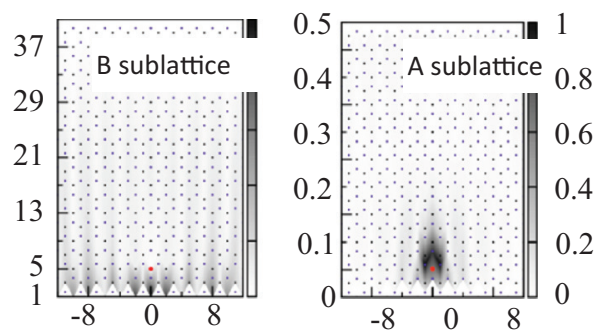

(b)

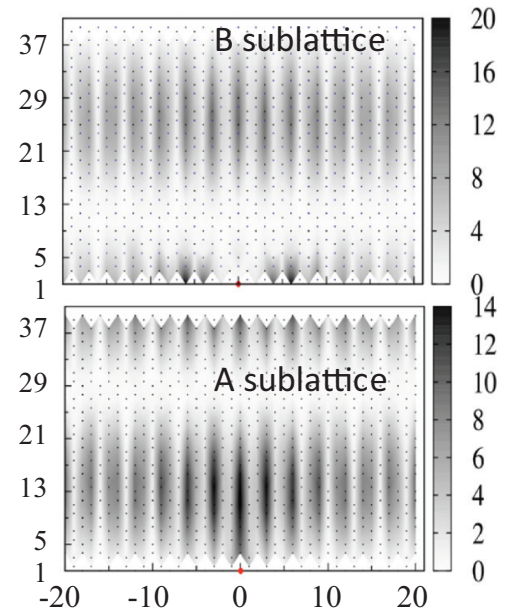

FIG. 6. (Color online) LDOS of a zigzag nanoribbon with a single defect at the sublattice $A$ at the resonant energy corresponding to the transmission $\operatorname{dip}(E=0.216544)$. Defect position is (a) $n=1$, (b) $n=5$ (site numbers at the $x$ and $y$ axis and the numbering of the defect position are the same as in the previous figures). The ribbon's width is $M=40$ sites.

the ribbon the amplitudes of the wave functions on the $A$ and $B$ sublattices are rather similar. As a result, a coupling between the extended states and the localized states at the defects residing at both the $A$ and $B$ sublattices is also similar, which leads to a similar width of the resonant dips.

Let us now proceed to the case of zBGN; see Fig. 5(b). The quasibound state of a defect in bilayer graphene is localized in the layer where the defect is situated and has the same structure as the localized state in monolayer graphene. The extended state of $\mathrm{zBGN}$ in each layer has a structure which is very similar to that of zGNR. ${ }^{18,41}$ Because the structures of both the localized state and the extended state in $\mathrm{zBGN}$ and zGNR are similar, the behavior of the transmission coefficient of zBGN is practically the same as that of zGNR; cf. Figs. 5(a) and $5(\mathrm{~b})$.

Let us now consider aGNR. We first note that a defect in one of the sites in rows $3,6,9, \ldots$ leaves the transmission probability unchanged [ $T=1$ in Fig. 5(c)]. This is because the LDOS of the nanoribbon vanishes at these sites, and therefore the defect does not distort the wave function of the ribbon; see Fig. 4(d). A defect in one of the sites in rows 1, 2, 4, 5, .. leads to a zero-energy resonant dip. For the explanation of its origin, consider a defect, say, in row 4 belonging to the $A$ sublattice or in row 5 belonging to the $B$ sublattice. They are surrounded by sites from the opposite sublattices, two of which have a 
high LDOS; see Fig. 4(d). This means that the localized state will not be very distorted and its resonant energy will remain practically the same as for an infinite graphene sheet. Note that the width of the state is essentially the same for all the defect positions except for those closest to the ribbon's edge. As the wave function of the extended state alternates between zero and a constant value across the ribbon, its overlap with the localized state around the defect does not change much when one moves the defect across the ribbon. However, because the extended state vanishes outside the ribbon, the overlap between states will be smaller for the defect at the edge, which leads to a narrower resonance; see Fig. 5(c).

Finally, let us consider the case of aBGN; see Fig. 5(d). As for the case of aGNR, a defect in sites with a vanishing LDOS does not affect the transmission probability (rows 3, 6, $9, \ldots)$. In contrast to aGNR, the transmission probability of aBGN behaves qualitatively different depending on whether a defect is on the $A$ or $B$ sublattice. A defect in one of the sites in rows $1,2,4,5, \ldots$ in sublattice $A$ leads to a broad and shallow resonance dip at the energy $E \approx 0$. A defect in the same rows but at sublattice $B$ corresponds to dips with transmission $T=0$ and has a resonance energy significantly shifted from zero. To explain the origin and features of the resonances, let us focus on a representative case of a defect at sublattice $B$ in row 5 . It is surrounded by sites from sublattice $A$, all with low (or zero) LDOS; see Fig. 4(e). As discussed above for the case of $\mathrm{zGNR}$, this would lead to a significant distortion of the wave function of the localized state and a shifting of the resonant energy from $E=0$ to higher values. However, in contrast to zGNR, the energy and the width of the resonance dip for aBGN remain practically unchanged when the defect is moved toward the middle of the ribbon. This is because the LDOS of aBGN alternates between zero and the same constant value across the ribbon, consequently it distorts the localized state around different sites in the same way, and the overlap remains the same. Consider now a defect in row 4 but at sublattice $A$. Such a defect has two neighbors with a high LDOS; see Fig. 4(e). As a result, similarly to the cases discussed above, the localized state remains practically not distorted, which leads to a resonant dip with a resonant state with energy $E \approx 0$.

Let us finally note that the properties of resonant states, such as the transmission minimum, the resonance width, and the energy, depend on the defect strength. For example, an increase in the on-site potential strength leads to a decrease in the transmission minimum for the zero-energy resonant states in armchair nanoribbons.

\section{Effect of many defects}

Having understood the effect of a single defect on the conductance of monolayer and bilayer graphene nanoribbons, let us now turn to the case of many defects forming disordered edges. We consider two models of the disordered edge. In

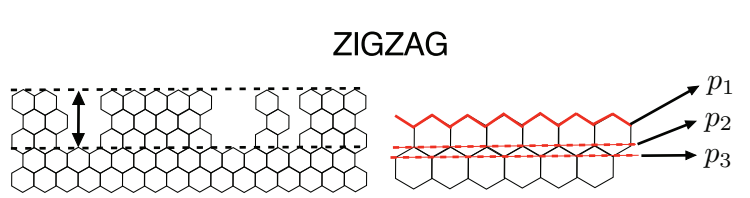

(a) zGNR

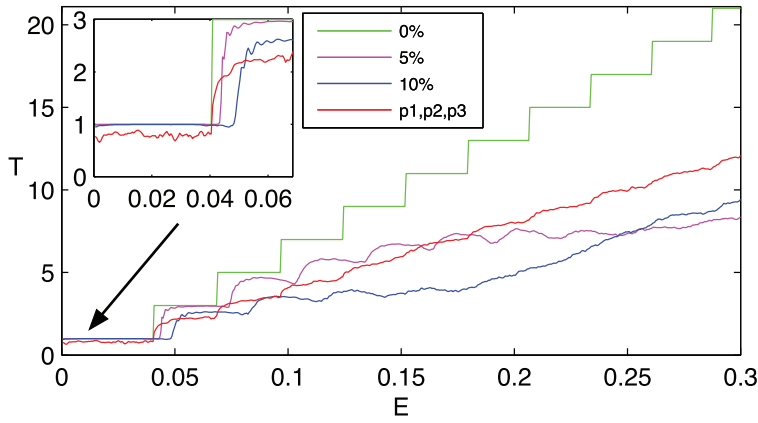

(c) zBGN

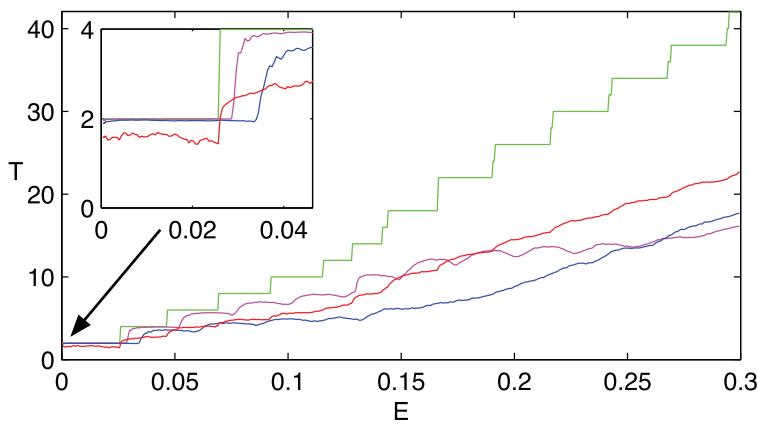

ARMCHAIR

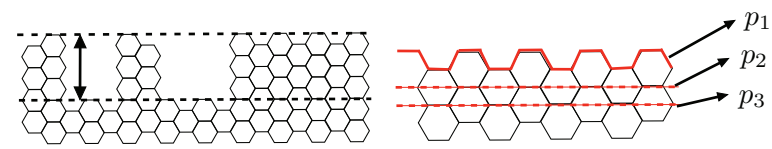

(b) aGNR

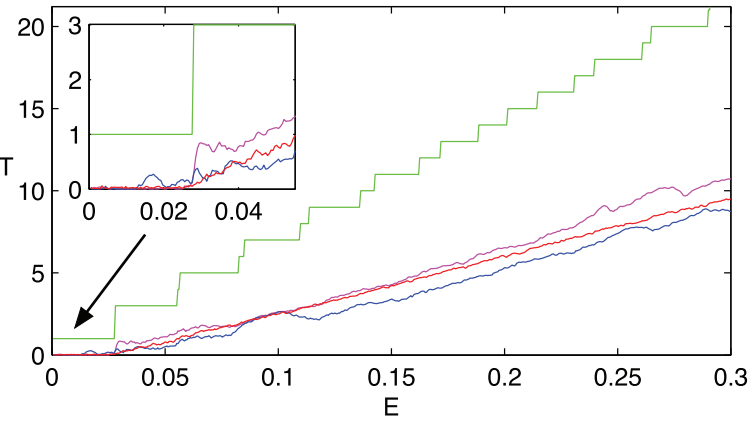

(d) aBGN

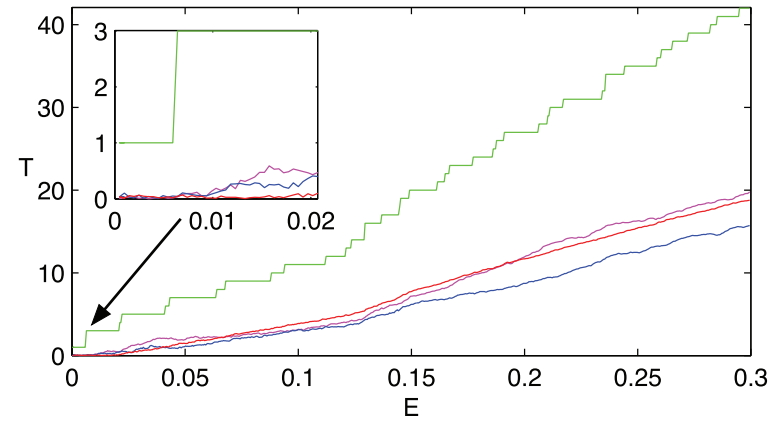

FIG. 7. (Color online) Transmission of monolayer and bilayer nanoribbons with disordered edges. (a) zGNR, (b) aGNR, (c) zBGN, and (d) aBGN. Top insets illustrate two models of disordered edges used in the present study. Simulations are averaged over 50 realizations. 
the first model, all the edge atoms covered by rectangular areas of random lengths and constant depths (of 5\% and $10 \%$ of the ribbon width) are removed, as shown in Fig. 7. Such a model apparently preserves the edge topology (i.e., zigzag or armchair) in the direction of transport. In the second model, we follow Ref. 18 and introduce a disordered edge by removing atoms from the outermost row (indicated by the red solid line in the insets) with probability $p_{1}=0.5$, followed by removing atoms from the second and third rows with conditional probabilities $p_{2}=0.5$ and $p_{3}=0.5$ if at least one of the adjacent atoms from the previous row is absent.

Figure 7 shows the transmission probability of monolayer and bilayer nanoribbons in the energy interval spanning both few- and many-mode regimes. For both models of disorder, the transmission of monolayer and bilayer nanoribbons of the same edge topology is rather similar, even though the transmission of bilayer nanoribbons is somehow lower than that of monolayer ones. This is consistent with the case of a ribbon with a single defect, where the corresponding transmission of monolayer and bilayer nanoribbons is also rather similar; see Figs. 2 and 3.

Let us now discuss differences and similarities in the transmission of zigzag and armchair nanoribbons. In the single- and few-mode regime, the conductance of both aGNR and $\mathrm{aBGN}$ is strongly suppressed. Note that even with a single defect, the conductance of armchair nanoribbons shows a noticeable suppression; see Figs. 2(b), 2(d), 3(b), and 3(d). With many defects this suppression adds up, leading to the formation of a pronounced transport gap of zero conductance. In contrast, in a few-, and especially in the single-mode regime, the conductance of zigzag ribbons is affected very little. This behavior can also be traced to the corresponding behavior of zigzag nanoribbons with a single defect, the conductance of which remains unchanged in comparison to the ideal case, provided that the defect is situated not far from the edge (Sec. III A). It is noteworthy that for the disorder preserving the edge topology, the conductance of both zGNR and zBGN in a single-mode regime remains practically unchanged in comparison to the ideal case.

In a many-mode regime, the difference in conductance between zigzag and armchair ribbons practically disappears; cf. Figs. 7(a) and 7(b) and Figs. 7(c) and 7(d). As discussed in Sec. III A, the distance to the edge for which the conductance of zigzag ribbons is not affected by the presence of the defect shrinks as the energy of the electrons increases [see Fig 3, right panels in (a)-(d)]. Thus, for high energies the armchair and zigzag ribbons become equally sensitive to the edge disorder.

\section{CONCLUSIONS}

We have studied the transmission properties of monoand bilayer graphene nanoribbons with defects, focusing on the role of edge termination (zigzag versus armchair). Using the standard tight-binding model of $p$-orbital electrons on a hexagonal lattice, we have developed an analytical approach based on the Green's function technique and the Dyson equation for calculation of the transmission coefficient of monolayer graphene nanoribbons with a single short-range defect. Calculation of the conductance in monolayer graphene nanoribbons with many defects and calculations for bilayer graphene nanoribbons is performed numerically on the basis of the tight-binding recursive Green's function technique. The principal conclusions of our work can be summarized as follows:

(i) For the case of the zigzag edge termination, both monolayer and bilayer nanoribbons in a single- and a fewmode regime remain practically insensitive to defects situated close to the edges. This remarkable behavior is related to the effective boundary condition at the zigzag edges which do not couple valleys, thus prohibiting intervalley scattering due to short-range defects situated close to the edges. In contrast, the armchair edges mix the valleys; as a result, the conductance of both monolayer and bilayer nanoribbons is strongly affected by even a small defect concentration at the edges.

(ii) For higher electron energies in the many-mode regime, the difference of the transmission between the armchair and zigzag ribbons diminishes, and for sufficiently high defect concentration they become equally sensitive to the edge disorder.

(iii) Both monolayer and bilayer nanoribbons with a shortrange defect show resonant features in the lowest energy mode. Resonances are identified to be of Fano type and emerge from the interference between the quasibound localized state around the defect and the extended state in the ribbon. We consider four different cases of a defect in (a) zGNR, (b) zBGN, (c) aGNR, and (d) aBGN. We discuss in detail how the interplay between the defects position at different sublattices in the ribbons, the defect distance to the edge, and the structure of the extended states in ribbons with different edge termination influence the width and the energy of Fano resonances.

\section{ACKNOWLEDGMENTS}

The authors thank T. Heinzel, Hengyi Xu, and A. A. Shylau for help with the programming code for the numerical calculations. I.V.Z. and J.R. acknowledge a collaborative grant from the Swedish Institute.

\section{APPENDIX: TRANSVERSE MODE WAVE FUNCTIONS}

The transverse mode wave function in aGNR is

$$
\begin{gathered}
\chi_{v, \kappa}^{\mathrm{aGNR}}(n, r)=\frac{1}{\sqrt{2(N+1)}} \sin \left(\xi_{v} n\right), \\
\chi_{v, \kappa}^{\mathrm{aGNR}}(n, \rho)=\frac{-1}{\sqrt{2(N+1)}} \frac{\phi\left(\kappa, \xi_{v}\right)}{E\left(\kappa, \xi_{v}\right)} \sin \left[\xi_{v}\left(n-\frac{1}{2}\right)\right],
\end{gathered}
$$

$$
\begin{gathered}
\chi_{v, \kappa}^{\mathrm{aGNR}}(n, \lambda)=\frac{-1}{\sqrt{2(N+1)}} e^{-i \frac{\kappa}{2}} \sin \left[\xi_{v}\left(n-\frac{1}{2}\right)\right], \\
\chi_{v, \kappa}^{\mathrm{aGNR}}(n, l)=\frac{1}{\sqrt{2(N+1)}} e^{-i \frac{\kappa}{2}} \frac{\phi\left(\kappa, \xi_{v}\right)}{E\left(\kappa, \xi_{v}\right)} \sin \left(\xi_{v} n\right) .
\end{gathered}
$$

For zGNR, taking into account Eq. (12) we have

$$
\chi_{v, \xi}^{\mathrm{zGNR}}(n, r)=C_{\nu, \xi}^{\mathrm{zGNR}} \sin \left(\kappa_{\nu} n\right),
$$




$$
\begin{gathered}
\chi_{\nu, \xi}^{\mathrm{ZGNR}}(n, \rho)=s_{1}(-1)^{\nu} C_{\nu, \xi}^{\mathrm{ZGNR}} e^{-i \frac{\xi}{2}} \sin \left[\kappa_{v}\left(N+\frac{1}{2}-n\right)\right], \\
\chi_{\nu, \xi}^{\mathrm{ZGNR}}(n, \lambda)=-C_{\nu, \xi}^{\mathrm{ZGNR}} e^{-i \frac{\xi}{2}} \sin \left[\kappa_{\nu}\left(n-\frac{1}{2}\right)\right], \\
\chi_{\nu, \xi}^{\mathrm{ZGNR}}(n, l)=s_{1}(-1)^{v+1} C_{\nu, \xi}^{\mathrm{ZGNR}} \sin \left[\kappa_{v}(N+1-n)\right],
\end{gathered}
$$

where

$$
C_{\nu, \xi}^{\mathrm{ZGNR}}=\left\{2 N-\frac{\sin \left(\kappa_{\nu} N\right)}{\sin \left(\frac{\kappa_{v}}{2}\right)} \cos \left[\kappa_{\nu}\left(N+\frac{1}{2}\right)\right]\right\}^{-\frac{1}{2}}
$$

is the normalization factor. It should be noted that for both wave functions $\chi_{v, k}^{\mathrm{aGNR}}$ and $\chi_{v, \xi}^{\mathrm{zGNR}}$, given by Eqs. (A1)-(A4) and (A5)-(A8), the following equality holds: $\chi_{v, \kappa_{\|}}^{\sigma *}\left(\ell_{\perp}\right)=\chi_{v,-\kappa_{\|}}^{\sigma}\left(\ell_{\perp}\right)$. *anna.orlof@liu.se

†Julius.ruseckas@tfai.vu.lt

†igor.zozoulenko@liu.se

${ }^{1}$ K. S. Novoselov, A. K. Geim, S. V. Morozov, D. Jiang, Y. Zhang, S. V. Dubonos, I. V. Grigorieva, and A. A. Firsov, Science 306, 666 (2004).

${ }^{2}$ M. Y. Han, B. Özyilmaz, Y. Zhang, and P. Kim, Phys. Rev. Lett. 98, 206805 (2007).

${ }^{3}$ F. Molitor, A. Jacobsen, C. Stampfer, J. Güttinger, T. Ihn, and K. Ensslin, Phys. Rev. B 79, 075426 (2009).

${ }^{4}$ X. Li, X. Wang, L. Zhang, S. Lee, and H. Dai, Science 319, 1229 (2008).

${ }^{5}$ D. V. Kosynkin, A. L. Higginbotham, A. Sinitskii, J. R. Lomeda, A. Dimiev, B. K. Price, and J. M. Tour, Nature (London) 458, 872 (2009).

${ }^{6}$ J. Cai, P. Ruffieux, R. Jaafar, M. Bieri, T. Braun, S. Blankenburg, M. Muoth, A. P. Seitsonen, M. Saleh, X. Feng, K. Müllen, and R. Fasel, Nature (London) 466, 470 (2010).

${ }^{7}$ X. Jia, J. Campos-Delgado, M. Terrones, V. Meuniere, and M. S. Dresselhaus, Nanoscale 3, 86 (2011).

${ }^{8}$ Y.-M. Lin, V. Perebeinos, Z. Chen, and P. Avouris, Phys. Rev. B 78, 161409 (2008).

${ }^{9}$ J. Davies, The Physics of Low-Dimensional Semiconductors (Cambridge University Press, Cambridge, 1998).

${ }^{10}$ D. A. Areshkin, D. Gunlycke, and C. T. White, Nano Lett. 7, 204 (2007).

${ }^{11}$ D. Gunlycke, D. A. Areshkin, and C. T. White, Appl. Phys. Lett. 90, 142104 (2007).

${ }^{12}$ D. Querlioz, Y. Apertet, A. Valentin, K. Huet, A. Bournel, S. GaldinRetailleau, and P. Dollfus, Appl. Phys. Lett. 92, 042108 (2008).

${ }^{13}$ A. Lherbier, B. Biel, Y.-M. Niquet, and S. Roche, Phys. Rev. Lett. 100, 036803 (2008).

${ }^{14}$ M. Evaldsson, I. V. Zozoulenko, H. Xu, and T. Heinzel, Phys. Rev. B 78, 161407 (2008).

${ }^{15}$ E. R. Mucciolo, A. H. Castro Neto, and C. H. Lewenkopf, Phys. Rev. B 79, 075407 (2009).

${ }^{16}$ S. Ihnatsenka and G. Kirczenow, Phys. Rev. B 80, 201407 (2009).

${ }^{17}$ A. Cresti and S. Roche, Phys. Rev. B 79, 233404 (2009).

${ }^{18}$ H. Xu, T. Heinzel, and I. V. Zozoulenko, Phys. Rev. B 80, 045308 (2009).

${ }^{19}$ J. Wurm, M. Wimmer, and K. Richter, Phys. Rev. B 85, 245418 (2012).

${ }^{20}$ F. Libisch, S. Rotter, and J. Burgdörfer, New J. Phys. 14, 123006 (2012).

${ }^{21}$ K. Wakabayashi, J. Phys. Soc. Jpn 71, 2500 (2002).
${ }^{22}$ Y.-J. Xiong and X.-L. Kong, Physica B 405, 1690 (2010).

${ }^{23}$ D. A. Bahamon, A. L. C. Pereira, and P. A. Schulz, Phys. Rev. B 82, 165438 (2010)

${ }^{24}$ J. C. Meyer, A. K. Geim, M. I. Katsnelson, K. S. Novoselov, T. J. Booth, and S. Roth, Nature (London) 446, 60 (2007).

${ }^{25}$ F. Schedin, A. K. Geim, S. V. Morozov, E. W. Hill, P. Blake, M. I. Katsnelson, and K. S. Novoselov, Nat. Mater. 6, 652 (2007).

${ }^{26}$ L. G. Cancado, M. A. Pimenta, B. R. A. Neves, M. S. S. Dantas, and A. Jorio, Phys. Rev. Lett. 93, 247401 (2004).

${ }^{27}$ C. Park, H. Yang, A. J. Mayne, G. Dujardin, S. Seo, Y. Kuk, J. Ihm, and Gunn Kim, Proc. Natl. Acad. Sci. (USA) 108, 18622 (2011).

${ }^{28} \mathrm{X}$. Chen, H. Wan, K. Song, D. Tang, and G. Zhou, Appl. Phys. Lett. 98, 263103 (2011); X. Chen, K. Song, B. Zhou, H. Wang, and G. Zhou, ibid. 98, 093111 (2011).

${ }^{29}$ K. Wakabayashi, Y. Takane, and M. Sigrist, Phys. Rev. Lett. 99, 036601 (2007)

${ }^{30}$ L. R. F. Lima, F. A. Pinheiro, R. B. Capaz, C. H. Lewenkopf, and E. R. Mucciolo, Phys. Rev. B 86, 205111 (2012).

${ }^{31}$ K. Wakabayashi, M. Fujita, H. Ajiki, and M. Sigrist, Phys. Rev. B 59, 8271 (1999).

${ }^{32}$ L. P. Zârbo and B. K. Nikolić, Europhys. Lett. 80, 47001 (2007).

${ }^{33}$ L. Brey and H. A. Fertig, Phys. Rev. B 73, 195408 (2006).

${ }^{34}$ T. Ando and T. Nakanishi, J. Phys. Soc. Jpn. 67, 1704 (1998).

${ }^{35}$ H. Xu, T. Heinzel, M. Evaldsson, and I. V. Zozoulenko, Phys. Rev. B 77, 245401 (2008).

${ }^{36}$ A. H. Castro Neto, F. Guinea, N. M. R. Peres, K. S. Novoselov, and A. K. Geim, Rev. Mod. Phys. 81, 109 (2009).

${ }^{37}$ J. Nilsson, A. H. Castro Neto, F. Guinea, and N. M. R. Peres, Phys. Rev. B 78, 045405 (2008).

${ }^{38}$ A. Ferreira, J. Viana-Gomes, J. Nilsson, E. R. Mucciolo, N. M. R. Peres, and A. H. Castro Neto, Phys. Rev. B 83, 165402 (2011).

${ }^{39}$ T. O. Wehling, S. Yuan, A. I. Lichtenstein, A. K. Geim, and M. I. Katsnelson, Phys. Rev. Lett. 105, 056802 (2010).

${ }^{40}$ A. Onipko, Phys. Rev. B 78, 245412 (2008).

${ }^{41}$ J. Ruseckas, G. Juzeliunas, and I. V. Zozoulenko, Phys. Rev. B 83, 035403 (2011).

${ }^{42}$ L. Brey and H. A. Fertig, Phys. Rev. B 73, 235411 (2006).

${ }^{43}$ A. R. Akhmerov and C. W. J. Beenakker, Phys. Rev. B 77, 085423 (2008).

${ }^{44}$ D. R. da Costa, A. Chaves, G. A. Farias, L. Covaci, and F. M. Peeters, Phys. Rev. B 86, 115434 (2012).

${ }^{45}$ E. N. Economou, Green's Functions in Quantum Physics (Springer, Heidelberg, 2006).

${ }^{46}$ S. Datta, Electronic Transport in Mesoscopic Systems (Cambridge University Press, Cambridge, 1997). 
${ }^{47}$ V. M. Pereira, F. Guinea, J. M. B. Lopes dos Santos, N. M. R. Peres and A. H. Castro Neto, Phys. Rev. Lett. 96, 036801 (2006).

${ }^{48}$ U. Fano, Phys. Rev. 124, 1866 (1961).

${ }^{49}$ W. Porod, Z. A. Shao, and C. S. Lent, App. Phys. Lett. 61, 1350 (1992).
${ }^{50}$ W. Porod, Z. A. Shao, and C. S. Lent, Phys. Rev. B 48, 8495 (1993).

${ }^{51}$ P. J. Price, Appl. Phys. Lett. 62, 289 (1993).

${ }^{52} \mathrm{H}$. Xu, T. Heinzel, M. Evaldsson, S. Ihnatsenka, and I. V. Zozoulenko, Phys. Rev. B 75, 205301 (2007). 